\title{
Serum soluble lectin-like oxidized low-density lipoprotein receptor-1 levels in patients with restless legs syndrome
}

\author{
Halac $\mathrm{G}^{1}$, Kilic $\mathrm{E}^{2}$, Cikrikcioglu $\mathrm{MA}^{3}$, Celik $\mathrm{K}^{3}$, Toprak-Erek $\mathrm{A}^{4}$, Keskin $\mathrm{S}^{2}$, Gultepe $\mathrm{I}^{3}$, Celik $\mathrm{RS}^{3}$, \\ Ozaras $\mathrm{N}^{5}$, Yildiz A ${ }^{6}$, Aydin $\mathrm{S}^{7}$, Akan $\mathrm{O}^{8}$, Karatoprak $\mathrm{C}^{3}$, Sekin $\mathrm{Y}^{3}$, Asil $\mathrm{T}^{1}$ \\ Department of Neurology, Bezmi Alem Vakif University, Faculty of Medicine, Istanbul, Turkey. \\ halacdr@yahoo.com
}

\begin{abstract}
OBJECTIVE: The aim of this study was to assess the predisposition for atherosclerosis in patients with RLS through serum SLOX-1 (serum Lectin-Like Oxidized Low-Density Lipoprotein Receptor-1) measurements. BACKGROUND: Recent epidemiological studies have suggested an association of RLS with certain chronic conditions such as diabetes mellitus (DM), obesity, hypertension (HT), and hyperlipidemia. LOX-1 is expressed in endothelial cells, macrophages, and in smooth muscle cells under the effect of proatherogenic conditions. METHODS: This study was a prospective, cross-sectional, case-controlled. We measured the serum sLOX-1 levels in 37 restless legs syndrome patients and 38 controls.

RESULTS: Serum SLOX-1 level was significantly lower in the patient group. The two groups were similar in glucose, HbA1c, creatinine, LDL cholesterol, TG, HDL, total protein, albumin, AST, ALT, GGT, ALP, HGB, HCT, MCV, transferrin saturation rate (TSR), ferritin, CRP, TSH, FT4, FT3, B12, and folic acid levels. Also the two groups were similar with respect to age at menarche, number of previous births, number of abortions and/or curettage, total duration of breastfeeding, percentage of patients in menopause, and age at menopause.

CONCLUSION: Our results may suggest a lower atherosclerotic risk among RLS patients as compared to the general population (Tab. 3, Ref. 33). Text in PDF www.elis.sk.

KEY WORDS: restless legs syndrome, atherosclerosis, sLOX-1.
\end{abstract}

\section{Introduction}

Restless legs syndrome (RLS) is a disorder characterized by a distressing urge to move and abnormal sensations that are difficult to depict in the legs (and sometimes in the arms) that tend to be relieved by movements. The symptoms get worse during rest, and are particularly distressing in the evening or night hours, frequently leading to a disruption of sleep in terms of initiating or continuing the sleep. It is followed by a fluctuating, progressive chronic clinical course with a negative impact on the quality of life (1). The reported prevalence rate among adults is 5 to $20 \%$ (2) with a female to male ratio of approximately 2 to 1 (3). The

${ }^{1}$ Department of Neurology, Bezmi Alem Vakif University, Faculty of Medicine, Istanbul, Turkey, ${ }^{2}$ Department of Clinical Biochemistry, Bezmi Alem Vakif University, Faculty of Medicine, Istanbul, Turkey, ${ }^{3}$ Department of Internal Medicine, Bezmi Alem Vakif University, Faculty of Medicine, Istanbul, Turkey, ${ }^{4}$ Department of Biochemistry, Istanbul Medeniyet University, Faculty of Medicine, Istanbul, Turkey, ${ }^{5}$ Department of Physical Medicine and Rehabilitation, Bezmi Alem Vakif University, Faculty of Medicine, Istanbul, Turkey, ${ }^{6}$ Department of Cardiology, Dicle University, Faculty of Medicine, Diyarbakir, Turkey, ${ }^{7}$ Yedikule Chest Disease and Thorax Surgery Education and Research Hospital, Department of Neurology, Istanbul, Turkey, and ${ }^{8}$ Okmeydani Education and Research Hospital, Department of Neurology, Istanbul, Turkey

Address for correspondence: G. Halac, Bezm-i Alem Vakif University, Medical Faculty, Department of Neurology, 34093 Fatih Istanbul, Turkey. Phone: +90.212 .5332326 , Fax: +90.212 .6217580$ pathogenesis is thought to involve a primary dopaminergic dysfunction on the background of a genetic predisposition and iron deficiency anemia is frequently considered to trigger this dopaminergic dysfunction $(3,4)$.

Recent epidemiological studies have suggested an association of RLS with certain chronic conditions such as diabetes mellitus (DM), obesity, hypertension (HT), and hyperlipidemia (5, 6). Also, an increased risk of cardiovascular diseases (CVD) has been reported in these patients $(6,7,8,9,10,11)$. On the other hand, other studies have failed to detect such an association between RLS and CVD $(12,13,14,15)$. Furthermore, a lower rate of atherosclerotic progression as compared to general population was reported by others, implying a protective effect of RLS on atherosclerosis (16, 17). The exact nature of the associations between RLS and these related conditions is currently unknown.

LOX-1(Lectin-Like Oxidized Low-Density Lipoprotein Receptor-1) is a type II membrane protein that belongs to C-type lectin family (18). It is expressed in endothelial cells, macrophages, and in smooth muscle cells under the effect of the pro-inflammatory stimulation by TNF-alpha, CRP, IL-2, angiotensin II, or endothelin, or in proatherogenic conditions such as DM, HT, and HL. The extracellular portion of LOX-1 receptor can be cleaved proteolytically and may be released in a soluble form. In this study, our aim was to assess the predisposition for atherosclerosis in patients with RLS through serum sLOX-1 measurements. To our knowledge, sLOX-1 levels have not been evaluated in patients with RLS yet. 


\section{Materials and methods}

\section{Study design and study population}

This was a prospective, cross-sectional, case-controlled study. A total of 37 patients with a diagnosis of primary RLS who met the inclusion and exclusion criteria and who were followed-up at the outpatient units of the departments of neurology or internal medicine, Bezmialem Vakif University Hospital between 2014 and March 2015 were included in this study after providing written informed consent. The control group consisted of a total of 38 BMI-matched women without RLS who provided written informed consent for the study, met the exclusion and inclusion criteria and attended the same outpatient units during the same time period.

\section{Inclusion criteria}

Drug naïve women 18 years and older with a diagnosis of RLS with or without concomitant iron deficiency anemia were included in this study. Control group comprised of women $\geq 18$ years of age with or without iron deficiency anemia without diagnosis of RLS.

\section{Exclusion criteria}

Patients with conditions other than iron deficiency anemia that may lead to secondary RLS such as uremia, cancer, peripheral vascular disease, rheumatoid arthritis, hypothyroidism, polyneuropathy, lumbosacral radiculopathy, amyotrophic lateral sclerosis, myelopathy, multiple sclerosis, or poliomyelitis were excluded. Other exclusion criteria were pregnancy, use of neuroleptics or antidepressants, severe hyperlipidemia, ischemic cardiac disease, cerebrovascular disease, peripheral arterial disease, local and systemic infectious diseases, chronic hepatitis, transaminase levels exceeding 2 times the upper limit of the normal range, portal hypertension, collagen tissue disorders, hematological malignancy, hemolytic anemia, inflammatory bowel disease, psoriasis, sarcoidosis, alcoholism, and a history of drug abuse. Furthermore, patients receiving medications that can trigger restless leg syndrome such as antidepressants, antipsychotics, lithium, and calcium antagonists were also excluded.

\section{Patient assessments}

The past medical and family history as well as ongoing medical treatments were assessed and recorded. Physical examination was performed in all patients and controls and height, weight and blood pressure measurements were taken. Cigarette smoking, use of alcohol, previous pregnancy or abortion, and menopausal status were determined. Blood and urinalysis were performed for determining the comorbid conditions. If required imaging studies and electroneuromyography were also done.

The diagnosis of RLS was based on the International RLS Working Group criteria (19) and all patients met the four essential diagnostic criteria required to establish a diagnosis of RLS. The severity of RLS was assessed using IRLSSG Severity Scale (20), which is an assessment tool directing questions on the typical symptoms of RLS using 5 point scale (i.e. between 0 and 4 ). A total score of 0 to 10,11 to 20,21 to 30 , and 31 to 40 is indicative of mild, moderate, severe, and very severe RLS.

\section{Laboratory}

Sampling: The blood samples were obtained in the early morning after overnight fasting. Routine blood analyses were performed on the same days. Blood samples were collected for LOX-1 in gel separated tubes and centrifuged for 10 minutes at $1500 \mathrm{~g}$. Supernatant serum was removed into polypropylene plastic tubes. Serum samples were stored at $-80{ }^{\circ} \mathrm{C}$ until the time of analysis.

Blood analysis: A complete blood cell count (CBC) analysis was performed using a Sysmex XT 1800i apparatus (ROCHE-2011, Kobe, Japan). Biochemical assays were performed on a COBAS 8000 apparatus (ROCHE-2007, Tokyo, Japan) using kits specific for the COBAS-C system. The levels of thyroid hormones were examined using a Siemens Advia Centaur apparatus (Siemens-2006, Dublin, Ireland) by a chemiluminescent method using Advia Centaur kits (Advia-2013-Tarrytown, USA).

ELISA analysis: The concentrations of human soluble lectinlike oxidized low density lipoprotein receptor-1(sLOX-1) in serum were measured with enzyme linked immunosorbent assay (ELISA) kit, according to protocols provided by manufacturers (Human Slox-1 Elisa Kit Eastbiopharm; cat no: CK-E10765; lot no:20130724; Hangzhou-China). Multiskan FC® Microplate Photometer (Thermo Scientific; United States) was used for reading at $450 \mathrm{~nm}$. The results were expressed in $\mathrm{ng} / \mathrm{ml}$.

\section{Statistics}

Numerical variables are presented as means with standard deviations, and nominal variables are presented as ratios. The subjects who were recruited into the study were divided into the RLS and control groups. The nominal independent variables were compared between the groups using chi-square test. A onesample Kolmogorov-Smirnov test was performed to determine if the continuous (numerical) independent variables were normally distributed. Normally distributed independent continuous variables were compared using Student's t test, whereas not normally distributed independent continuous variables were compared between the groups using the Mann-Whitney U test. Bivariate correlations were sought. A two-tailed $p$ value of $<0.05$ was considered to be statistically significant.

\section{Ethical considerations}

The study protocol was approved by Bezmialem Vakif University Medical Faculty Ethics Committee, and all the subjects who were recruited into study gave informed consent. The study was performed in accordance with the principles set forth in 2009 Helsinki Declaration.

\section{Results}

A total of 75 female individuals, 37 patients with RLS and 38 controls were included in this study. The mean age and age range in the overall study population were $45.2 \pm 12.12$ years and $25-73$ years, respectively, with no significant age differences between the two groups (Tab. 1). The average BMI in the study population was $28.06 \pm 3.88 \mathrm{~kg} / \mathrm{m}^{2}$ (range: $20.5-35.80$ ) and it was similar to that in the control group (Table 1). The average duration of RLS 
Tab. 1. Demographic data in RLS and control subjects.

\begin{tabular}{|c|c|c|c|c|c|c|}
\hline \multirow{2}{*}{ Parameters } & \multicolumn{2}{|c|}{ RLS } & \multicolumn{2}{|c|}{ Control group } & \multirow[b]{2}{*}{$\mathrm{p}$ value } & \multirow{2}{*}{ Type of distribution } \\
\hline & mean & \pm sd & mean & $\pm \mathrm{sd}$ & & \\
\hline Age, years & 44.2 & \pm 10 & 46.3 & \pm 13.8 & 0.463 & ND \\
\hline BMI, $\mathrm{kg} / \mathrm{m}^{2}$ & 28.3 & \pm 3.7 & 27.8 & \pm 4 & 0.589 & ND \\
\hline Systolic blood pressure & 122,5 & \pm 13.4 & 124.7 & \pm 13.4 & 0.500 & ND \\
\hline Diastolic blood pressure & 70 & \pm 9 & 73 & \pm 10.2 & 0.218 & ND \\
\hline No of births. & 2.3 & \pm 1.1 & 2.8 & \pm 1.8 & 0.177 & ND \\
\hline No. of abortions/curettage & 0.97 & \pm 1.3 & 0.86 & \pm 1.2 & 0.940 & NND \\
\hline Age at menopause & 47,2 & 5.868 & 50.8 & 4.59 & 0.120 & ND \\
\hline Age at menarche & 13.8 & 1.417 & 12.6 & 1.125 & 0.432 & ND \\
\hline
\end{tabular}

ND - Normal distribution, NND - UnAbnormal distribution

was $52.82 \pm 61.35$ months (range: $12-360$ ), and the average RLS severity according to IRLLSS was $29.94 \pm 5.23$ (range: 17-39).

Also the two groups were similar with respect to age at menarche, number of previous births, number of abortions and/or curettage, total duration of breastfeeding, percentage of patients at menopause, and age at menopause (Tab. 1).

Also patients and controls were comparable in terms of blood pressure, proportion of patients with Type 2 DM, or HT, as well as the treatments given for hypertension or diabetes, and number of current and ex-smokers (Tab. 2).

Serum sLOX-1 level was significantly lower in the patient group (Tab. 3). The two groups were similar in glucose, HbAlc, creatinine, LDL cholesterol, TG, HDL, total protein, albumin, AST, ALT, GGT, ALP, HGB, HCT, MCV, transferrin saturation rate (TSR), ferritin, CRP, TSH, FT4, FT3, B12, and folic acid levels (p > 0.05) (Tab. 3).

A bivariate correlation analysis was performed to examine associations between SLOX-1 and LDL, TG, HDL, RLS severity, duration of RLS without treatment, BMI, age, ferritin and TSR. A negative correlation between the severity of RLS and serum sLOX-1 was found $(r=-0.288, p=0.012)$, while there were no significant correlations between sLOX-1 and LDL, TG, HDL, duration of RLS without treatment, BMI, age, ferritin, and TSR.

\section{Discussion}

Despite our limited understanding regarding the regulatory mechanisms for the release of sLOX-1, elevated serum levels of sLOX-1 assist in predicting the severity and prognosis of atherosclerotic vascular disease (21). Chen et al. showed that (22) endothelial LOX-1 expression occurs at very early stages of atherosclerosis. LOX-1 plays a role in endothelial activation for the development of atherosclerosis. LOX-1 acts like an endothelial sensor for the oxidized LDL in the vessel wall. When LOX-1 interacts with oxidized LDL a number of cellular and morphological changes occur as well as the stimulation of endothelial dysfunction, smooth muscle proliferation, and apoptosis. It is involved in the transformation of macrophages into bubble cells and in the formation and destabilization of the atherosclerotic plaque through the induction of platelets. Similar to troponin-T, it is also a biomarker for myocardial injury in acute coronary syndrome (23). In patients with diabetes mellitus, sLOX-1 serum levels have been found to be positively correlated with $\mathrm{HbA1c}$ (24). In postmenopausal obese women, serum SLOX-1
Tab. 2. Comorbid conditions, smoking habits, and medications in patient and control subjects.

\begin{tabular}{lccc}
\hline & $\begin{array}{c}\text { RLS Group } \\
(\mathrm{n}=37)\end{array}$ & $\begin{array}{c}\text { Control Group } \\
(\mathrm{n}=38)\end{array}$ & $\mathrm{p}$ value \\
\hline Smokers & 13 & 10 & 0.408 \\
Ex-smokers & 3 & 2 & 0.675 \\
Diabetes mellitus type 2 & 2 & 4 & 0.414 \\
Essential hypertension & 4 & 6 & 0.736 \\
Menopause & 9 & 13 & 0.347 \\
Metformin & 1 & 4 & 0.174 \\
Gliclazide & - & 1 & 0.327 \\
ACE inhibitor & - & 3 & 0.085 \\
Angiotensin receptor blocker & 2 & 3 & 0.666 \\
Beta- blocker & - & 2 & 0.157 \\
\hline
\end{tabular}

RLS - restless legs syndrome

levels show a positive correlation with BMI (25). Conversely, serum SLOX-1 levels are reduced by weight loss, and by treatment with angiotensin receptor blockers, statins, or antidiabetics (26). In this study, patients with RLS had lower serum sLOX-1 levels as compared to controls that were not significantly different from RLS patients in term of DM and HT incidence, use of antihypertensives or antidiabetics, or proportion of current or ex-smokers. Also number of births, number of abortions/curettage, duration of breastfeeding, and menopausal state were similar across the groups as well as the results of comprehensive biochemistry panel.

In cross-sectional studies by Ulfberg et al (8), Ohayon and Roth (9), and Winkelman et al (10) a total of 4000 patients between 18 and 64 years of age, 18.980 patients over 15 years of age, and 3433 patients were included, respectively. These studies examined whether a diagnosis of RLS was associated with an increased risk of cardiovascular disease, and all these three studies found a higher incidence of cardiovascular disease among patients with RLS. On the other hand, in the study by Winter et al $(13,4)$ involving 30,262 patients, no association between RLS and increased incidence of vascular events (major CVD, myocardial infarction, stroke, coronary revascularisation and CVD death) could be found. These epidemiological studies involving large sample populations, questionnaires completed by the patients or telephone calls were utilized to collect data rather than face-to-face interviews or examination, leading to a risk of identification of a certain number of individuals with a false positive diagnosis of RLS. Another major limitation of these studies is the failure to utilize data regarding the duration, incidence and severity of RLS for analyses. These large-scale epidemiological studies 
Tab. 3. Results of blood analyses in patient and control groups.

\begin{tabular}{|c|c|c|c|c|c|c|c|}
\hline \multirow[t]{2}{*}{ Parameters } & \multicolumn{2}{|c|}{$\begin{array}{l}\text { RLS Group } \\
(n=37)\end{array}$} & \multicolumn{2}{|c|}{$\begin{array}{l}\text { Control Group } \\
(n=38)\end{array}$} & \multirow{2}{*}{$\begin{array}{c}\text { Reference } \\
\text { value }\end{array}$} & \multirow[t]{2}{*}{$\mathrm{p}$ value } & \multirow{2}{*}{$\begin{array}{c}\text { Type of } \\
\text { distribution }\end{array}$} \\
\hline & mean & \pm sd & Mean & \pm sd & & & \\
\hline sLOX-1 & 2.04 & \pm 0.5 & 3.7 & \pm 2.9 & unknown & $0.008^{*}$ & NND \\
\hline Glucose, $\mathrm{mmol} / \mathrm{L}$ & 95.3 & \pm 14.3 & 99.9 & \pm 31.7 & $70-105$ & 0.538 & NND \\
\hline HbA1c, $\%$ & 6.3 & \pm 4.3 & 5.6 & \pm 0.6 & $4.8-5.9$ & 0.585 & NND \\
\hline Creatinine, mg/dl & 0.58 & \pm 0.1 & 0.64 & \pm 0.1 & $0.5-1.1$ & 0.166 & NND \\
\hline LDL-cholesterol, $\mathrm{mmol} / \mathrm{L}$ & 130.9 & \pm 33.4 & 132 & \pm 47.8 & $<130$ & 0.907 & ND \\
\hline Triglyceride, mmol/dL & 152,5 & \pm 96.4 & 117 & \pm 69.1 & $50-150$ & 0.077 & ND \\
\hline HDL-cholesterol, $\mathrm{mmol} / \mathrm{L}$ & 52.5 & \pm 13.9 & 58.9 & \pm 12.9 & $35-70$ & 0.049 & ND \\
\hline Total protein, g/L & 7.3 & \pm 0.3 & 7.3 & \pm 0.4 & $6.4-8.3$ & 0.674 & ND \\
\hline Albumin, $g / L$ & 4.4 & \pm 0.25 & 4.3 & \pm 0.2 & $3.4-4.8$ & 0.114 & ND \\
\hline AST, IU/L & 21.9 & \pm 9.7 & 20.8 & \pm 9.8 & $5-34$ & 0.667 & NND \\
\hline ALT, IU/L & 22.8 & \pm 14.5 & 23.8 & \pm 24.4 & $0-55$ & 0.903 & NND \\
\hline GGT, IU/L & 22 & \pm 16.3 & 18.8 & \pm 11.9 & $9-36$ & 0.274 & NND \\
\hline ALP, IU/L & 66.5 & \pm 17.1 & 70.4 & \pm 22.9 & $40-150$ & 0.420 & ND \\
\hline $\mathrm{Htc}, \%$ & 38.3 & \pm 4 & 38.4 & \pm 3.3 & $35.5-48$ & 0.958 & ND \\
\hline $\mathrm{MCV}, \mathrm{fL}$ & 82.6 & \pm 8.2 & 83.5 & \pm 4.9 & $80-97$ & 0.557 & ND \\
\hline TSR, $\%$ & 18.5 & \pm 13.6 & 22.35 & \pm 10.8 & $20-40$ & 0.191 & ND \\
\hline Iron, ug/dl & 65.7 & \pm 42.4 & 76.9 & \pm 34.2 & $37-145$ & 0.209 & ND \\
\hline Iron binding capacity, ug/dl & 378.7 & \pm 74.2 & 357.4 & \pm 52.8 & $250-425$ & 0.155 & ND \\
\hline Ferritin, ng/mL & 41.04 & \pm 83.5 & 32.4 & \pm 32.5 & $10-291$ & 0.735 & NND \\
\hline $\mathrm{CRP}, \mathrm{mg} / \mathrm{dL}$ & 0.6 & \pm 1.1 & 0.2 & \pm 0.2 & $<0.5$ & 0.618 & NND \\
\hline TSH, mIU/L & 2.25 & \pm 1.5 & 2.3 & \pm 1.5 & $0.3-4.9$ & 0.888 & ND \\
\hline Vitamin B12, pg/mL & 323 & \pm 111.6 & 384 & \pm 121.7 & $187-900$ & 0.260 & ND \\
\hline Folic acid & 9.03 & \pm 3.4 & 8.5 & \pm 3.8 & $5-31.4$ & 0.533 & ND \\
\hline
\end{tabular}

ND - Normal distribution, NND - Abnormal distribution, TSR - transferrin saturation rate

suggest that the association between RLS and hypertension and/or cardiovascular diseases is uncertain. In another cross-sectional study involving patients over 40 years of age from Iceland and Sweden, physical examination and biochemical tests were also performed, and no association was reported between RLS and CVD (15).

In a longitudinal study by $\mathrm{Li}$ et al (11), however, there was an increased incidence of non-fatal myocardial infarction in women with RLS who were followed-up for a minimum duration of 3 years. The observed association was independent of the known risk factors of CVD including age, cigarette smoking, physical activity, dietary patterns, BMI, use of antidepressants, history of HT, DM, high total cholesterol, shorter or longer duration of sleep, or snoring. On the other hand, a prospective study from the UK looking at the incidence of vascular events in RLS patients followed-up for 10 years, showed an association of RLS with stroke incidence, but not with ischemic heart disease (12). As the studies mentioned above suggest, cardiac or cerebral ischemic event risk in patients with RLS is either increased or unchanged.

Even studies reporting a protective effect for RLS against atherosclerosis are present. In a recent study by Park et al (16) RLS patients had significantly lower carotid intima-media thickness, which is a marker of atherosclerosis, as compared to controls; the authors concluded that patients with RLS may have a lower risk of atherosclerotic progression. In another condition potentially sharing similar pathophysiological mechanisms with RLS, i.e. Parkinson's disease (PD), although a lower risk of atherosclerosis and related cerebrovascular disease risk was reported, it is known that atherosclerosis risk may arise due to the development of secondary hyperhomocysteinemia resulting from L-dopa treatment in PD patients receiving such agents (27). A similar study found increased carotid intima-media thickness in PD patients receiving L-Dopa treatment (28), providing some support for our findings.

It is likely that functional and absolute deficiency of dopamine in the central nervous system may be associated with a decreased risk of atherosclerosis in RLS, although the exact pathophysiological mechanisms remain unidentified.

In a previous cross-sectional study by our team involving RLS patients, total oxidative state, arylestherase (ARE) and acetyl choline estherase (AChE) levels were higher, and lipid hydroperoxidase (LOOHs) levels were lower in these patients (29). Experimental and prospective studies suggest that ARE and PON may prevent the development of atherosclerosis (30), while LOOHs may contribute to the progression of atherosclerotic lesions (31). The results of our previous study examining the oxidative stress status in RLS patients are in line with our current findings.

Wahlin-Larsson et al (32) studied the morphology of the skeletal muscle in patients with RLS and showed an increased microvessel tortuosity in these individuals. The authors proposed that the disturbance of the balance between vascoconstriction and vasodilation may be responsible for the emergence of the symptoms and that re-establishment of this balance through the movements of the extremity may provide symptomatic relief. Increase in flow-induced laminar shear stress is a strong physiological stimulant with exercise-related atheroprotective effects. This may partly explain the lower sLOX levels in RLS levels, since RLS patients generally experience an increased frequency of extremity movements in night hours as well as the day hours. However, it is not clear whether this can be considered a type of exercise. We believe that 
the increased frequency of movements performed by RLS patients for the relief of their symptoms may play a role in lower SLOX-1 levels. However, to our knowledge, no studies supportive of this hypothesis have been reported until now. On the other hand, it is a well-established fact that aerobic exercise may prevent or delay the development of atherosclerosis (33). The negative correlation between SLOX-1 and RLS severity in our study may be indicative of the fact that movements associated with more severe RLS may have a role in the inhibition of SLOX-1 release.

A limitation of our study is its relatively smaller sample size.

\section{Conclusion}

To the best of our knowledge, this is the first study to report on serum sLOX-1 levels in patients with RLS that are known to exhibit increased expression during the early phase of atherosclerosis. Although we do not know the exact mechanisms through which sLOX-1 is reduced in RLS patients, our results may suggest a lower atherosclerotic risk among RLS patients as compared to the general population. Further cross-sectional studies examining SLOX-1 levels, larger samples and studies looking at the change in sLOX1 levels after treatment of restless leg syndrome are warranted.

\section{References}

1. Allen RP, Earley CJ. Restless legs syndrome: a review of clinical and pathophysiologic features. J Clin Neurophysiol 2001; 18: 128-147.

2. Kushida CA. Clinical presentation, diagnosis, and quality of life issues in restless legs syndrome. Am J Med 2007; 120: 4-12.

3. Hogl B, Kiechl S, Willeit J et al. Restlegs legs syndrome: a community-based study of prevelance, severity, and risk factors. Neurology 2005; 64: 1920-1924.

4. Allen R. Dopamine and iron in the pathophysiology of restless legs syndrome. Sleep Med 2004; 5 (4): 385-391.

5. 5.Baskol G, Korkmaz S, Erdem F et al. Assessment of nitricoxide, advanced oxidation protein products, malondialdehyde, and thiol levels in patients with restless legs syndrome Sleep Med 2012; 13: 414-418.

6. Innes KE, Selfe TK, Agarwal P. Restless legs syndrome and conditions associated with metabolic dysregulation, sympathoadrenaldysfunction, and cardiovascular disease risk: A systematic review. Sleep Med Rev 2012; 16: 309-339.

7. Walters AE, Rye DB. Review of the Relationship of Restless Legs Syndrome and Periodic Limb Movements in Sleep to Hypertension, Heart Disease, and Stroke. Sleep 2009; 32 (5): 589-597.

8. Ulfberg J, Nystrom B, Carter $\mathbf{N}$ et al. Prevalence of restless legs syndrome among men aged 18 to 64 years: an association with somatic disease and neuropsychiatric symptoms. Mov Disord 2001; 16: 1159-1163.

9. Ohayon MM, Roth T. Prevalence of restless legs syndrome and periodic limb movement disorder in the general population. J Psychosom Res 2002; 53: 547-554.

10. Winkelman JW, Finn L, Young T. Prevalence and correlates of restless legs syndrome symptoms in the Wisconsin Sleep Cohort. Sleep Med 2006; 7: 545-552.

11. LiY, WaltersAS, Chiuve SE et al. Prospective study of restşess legs syndrome and coronary heart disease among women. Circulation 2012; 126: 1689-1694.

12. Elwood P, Hack M, Pickering $\mathbf{J}$ et al. Sleep disturbance, stroke, and heart disease events: Evidence from the Caerphilly cohort. J Epid Comm Health 2006; 6069-6073.

13. Winter AC, Schürks M, Glynn RJ et al. Restless legs syndrome and risk of incident cardiovascular disease in woman and men; prospective cohort study. BMJ Open 2012; 2: e000866
14. Winter AC, Schürks M, Glynn RJ et al. Vascular risk factors, cardiovascular disease and restless legs syndrome in women. Am J Med 2013; 126 (3): $220-2276$

15. Benediktsdottir B, Janson C, Lindberg E et al. Prevelance of restless legs syndrome among adults in Iceland and Sweden: Lung function, comorbidity, ferritin, biomarkers and quality of life. Sleep Med 2010; 11: 1043-1048.

16. Park JH, Han SW, Baik JS. Carotid intima-media thickness in patients with idiopathic restless legs syndrome. Eur Neurol 2012; 67 (6): 321-325.

17. Jacobo K, Monge A, Ortiz-Panozo E et al. Abstract P074: Restless Legs Syndrome and Carotid Intima-Media Thickness in Women Circulation. Session Title: Sleep 2015; 131.

18. Pirillo A, Catapano AL. Soluble Lectin-Like Oxidized Low Density Lipoprotein Receptor-1 as a Biochemical Marker for Atherosclerosis-Related Diseases. Hindawi Publishing Corporation Disease Markers 2013; 35: 413-418.

19. Walters AS. Toward a better definition of the restless legs syndrome. The International Restless Legs Syndrome Study Group. Mov Disord 1995; 10: 634-642.

20. The International Restless Legs syndrome Study Group. Validation of the International Restless Legs Syndrome Study Group rating scale for restless legs syndrome. Sleep Med 2003; 4: 121-132.

21. Kume N, Kita T. Lectin-Like Oxidized Low-Density Lipoprotein Receptor-1 (LOX-1) in Atherogenesis. TCM 2001; 11: 23-25.

22. Chen M, Kakutani B M, Minami M et al. Increased Expression of Lectin like Oxidized Low Density Lipoprotein Receptor-1 in Initial Atherosclerotic Lesions of Watanabe Heritable Hyperlipidemic Rabbits. Arterioscler Thromb Vasc Biol 2000; 20: 1107-1115.

23. Hayashida K, Kume H, Murase T et al. Serum Soluble Lectin-Like Oxidized Low-Density Lipoprotein Receptor-1 Levels Are Elevated in Acute Coronary Syndrome A Novel Marker for Early Diagnosis. Circulation 2005; 112: $812-818$.

24. Tan KC, Shiu SW, Wong Y et al. Soluble lectin-like oxidized low density lipoprotein receptor-1 in type 2 diabetes mellitus. J Lipid Res 2008; 49 : 438-1444.

25. Brinkley TE, Wang $X$, Kume $\mathbf{N}$ et al. Caloric restriction, aerobic exercise training and soluble Lectin-like Oxidized LDL receptor-1 levels in owerweight and obese postmenopausal woman. Int J Obes 2011; 35 (6): 793-799.

26. Navarra T, Del Turco S, Beti S et al. The Lectin-like oxidized low-density lioprotein receptor-1 and its soluble form: Cardiovascular implications. J Atheroscler Thromb 2010; 17: 317-331.

27. Bostantjopoulou S, Katsarou Z, Frangia T et al. Endothelial function markers in parkinsonian patients with hyperhomocysteinemia. J Clin Neurosci 2005; 12 (6): 669-672.

28. Nakaso K, Yasui K, Kowa H et al. Hypertrophy of IMC of carotid artery in Parkinson's disease is associated with L-DOPA, homocysteine, and MTHFR genotype. J Neurol Sci 2003; 207: 19-23.

29. Cikrikcioglu MA, Hursitoglu M, Erkal $\mathbf{H}$ et al. Oxidative stress and autonomic nervous system functions in restless legs syndrome. Eur J Clin Invest 2011; 41 (7): 734-742.

30. Mackness B, Quarck R, Verreth W et al. Human paraoxonase-1 overexpression inhibits atherosclerosis in a Mouse model of metabolic syndrome. Arterioscler Thromb Vasc Biol 2006; 26: 1545-1550.

31. Leitinger $\mathbf{N}$. Cholesteryl ester oxidation products in atherosclerosis. Mol Aspects Med 2003; 24: 239-250.

32. Wahlin-Larsson BW, Kadi F, Ulfberg J et al. Skeleteal muscle morphology in patients with Restless Legs Syndrome. Eur Neurol 2007; 58: 133-137. 33. Walsh JH, Yong G, Cheetham C et al. Effects of exercise training on conduit and resistance vessel function in treated and untreated hypercholesterolaemic subjects. Eur Heart J 2003; 24: 1681-1689.

Received September 30, 2015. Accepted October 5, 2015. 